\title{
Diagnosis of Disease through Voice Recordings using Artificial Neural Networks
}

\author{
Karunanithi. D \\ Research Scholar \\ Manonmaniam Sundaranar University \\ Tirunelveli, India
}

\author{
Dr. Paul Rodrigues \\ Professor \\ King Khalid University \\ Saudi Arabia
}

\begin{abstract}
The main cause for the Parkinson's Disease is Neurodegenerative brain disorder. The process of impairment of brain cells is called neurodegeneration. Generally, Parkinson's Disease is diagnosed by clinical diagnosis method. Existing clinical methods are difficult for early diagnosing of Parkinson's Disease through Invasive or Non-Invasive method. Artificial Neural Network provides a way to differentiate and diagnose the Parkinson's Disease. Artificial Neural Network method helps people to diagnose Parkinson's Disease earlier and saves their lives. This proposed method proves to be better for early identification of disease. This method uses Feed Forward Back Propagation and trainlm function for producing more accuracy. Among the comparative classifications, four are chosen for highest accuracy. This method found to be best for early deduction of the disease with result accuracy of 98.53 and 99.44 percent training and testing respectively.
\end{abstract}

Keywords: Artificial Intelligence; Artificial Neural Network; Parkinson's Disease; Disease Diagnosis; Machine Learning

\section{INTRODUCTION}

Parkinson's Disease is a neurodegenerative brain disorder. A person's brain slowly stops producing a neurotransmitter called dopamine. The less secretion of dopamine leads to less control to regulate their movements, body and emotions. The brains cells neuron produces the dopamine. These neurons concentrate in a particular region of brain called the substantia nigra. Dopamine is a chemical carries information from the substantia nigra to other parts of the brain to control movements of a human body. When 60 to $80 \%$ of dopamine cells got damaged, the symptoms of Parkinson's Disease appears. This process of impairment of brain cells is called neurodegeneration [1].

As the statics shows more than 6.2 million peoples are affected with PD. Normally at the age of 60 this PD affects the people. James Parkinsons is the one who first identifies this disease and written a detailed article named "Shaking Palsy" in the year 1817. This disease also named as Parkinson's Disease after the discovery of the disease by James Parkinson [2].

There are five stages in PD. In Stage 1, Tremor occurs in the first stage. Normally a person cannot able to do the daily activities. Tremor affects one side of the body.

In Stage 2, Tremor and other movement systems affects both side of the body. Walking problem occurs.

In Stage 3, the loss of balance and slowness occurs.

In Stage 4, It is a severe stage where a person needs assistance to walk.
Stage 5 is the most advanced stage, Where the person cannot able to stand and walk. Wheel chair is needed or bed ridden [3].

As of now there is no cure for PD. Only through the symptoms we can identify the disease. Various signals, including ECG [4] Speech [5]-[8] and gait have been undertaken for diagnosis of PD. Voice signal recording is the earliest, easiest, non-invasive method for diagnosing $\mathrm{PD}[9]$. Most of the people suffer from speech disorders [10][11], this method will be considered as the reasonable way for deduction of $\mathrm{PD}[12][13]$.

This research is to identify and diagnose the PD with the PD datasets through ANN concept. Using this dataset the PD and healthy persons can be classified using the ANN which helps in easy way to diagnose the PD.

\section{MATERIALS}

The Parkinson's Dataset consist of 195 instances multivariate biomedical voice measurements of 31 people among which 23 are affected with PD. Each column in the table is a particular voice measure, and each row corresponds one of 195 voice recording from these individuals. Each Individual is opted to have 5 to 6 records for 23 different parameters. Status column is to denote the individual is affected with PD or healthy. Status column is set to ' 0 ' for healthy and ' 1 ' for PD affected.

Each row in the dataset consists of different occurrence of one voice recording. Each individual voice is recorded six times [14]. 
Table 1 Parkinson's Dataset Attributes

\begin{tabular}{|l|l|l|l|l|l|}
\hline No & Attribute Info. & No & Attribute Info. & No & $\begin{array}{c}\text { Attribute } \\
\text { Info. }\end{array}$ \\
\hline 1 & MDVP:Fo(Hz) & 9 & MDVP:Shimmer & 17 & Status \\
\hline 2 & MDVP:Fhi(Hz) & 10 & MDVP:Shimmer(dB) & 18 & RPDE \\
\hline 3 & MDVP:Flo(Hz) & 11 & Shimmer:APQ3 & 19 & DFA \\
\hline 4 & MDVP:Jitter(\%) & 12 & Shimmer:APQ5 & 20 & Spread1 \\
\hline 5 & MDVP:Jitter(Abs) & 13 & MDVP:APQ & 21 & Spread2 \\
\hline 6 & MDVP:RAP & 14 & Shimmer:DDA & 22 & D2 \\
\hline 7 & MDVP:PPQ & 15 & NHR & 23 & PPE \\
\hline 8 & Jitter:DDP & 16 & HNR & & \\
\hline
\end{tabular}

MDVP:Fo(Hz) - Average vocal fundamental frequency

MDVP:Fhi(Hz) - Maximum vocal fundamental frequency

MDVP:Flo(Hz) - Minimum vocal fundamental frequency

MDVP:Jitter(\%),MDVP:Jitter(Abs),MDVP:RAP,MDV P:PPQ, Jitter:DDP - Several measures of variation in fundamental frequency

MDVP:Shimmer,MDVP:Shimmer(dB),Shimmer:APQ3 ,Shimmer:APQ5,MDVP:APQ,Shimmer:DDA - Several measures of variation in amplitude

NHR,HNR - Two measures of ratio of noise to tonal components in the voice

Status - Health status of the subject (one) - Parkinson's, (zero) - healthy

RPDE, D2 - Two nonlinear dynamical complexity measures

DFA - Signal fractal scaling exponent

Spread1, Spread2, PPE - Three nonlinear measures of fundamental frequency variation [14]

\section{METHODS}

Artificial Neural Network works in the way the human neurological system works. As humans learn by examples the ANN also learn by examples. A human brain consists of largely connected elements called neurons. A network consists of group of interconnected neurons. ANN is capable of Machine Learning, Pattern Recognition, Adaptive Learning, Self-Organization, Real Time Operation and Fault Tolerance. Neural Network process the information in a similar way the as the human brain does. The Neural Network composed of largely interconnected neurons which works in parallel to solve a problem. NN cannot be preprogrammed, as it learns by example. The Neurons can be Single Input
Neuron, Multiple Input Neuron and a complex Neuron consists of Multiple Layers. The Neuron model works in a way that input is given with the weight added to it and it is processed in the neuron with a transfer function and desired output will occur. A notation for single neuron can be written as

$a=f(w p+b)$

(1)

where $\mathrm{b}$ is the biased input.

A Multi Layered Neuron (3 Layers) can be notation can be given as

a $3=\mathrm{f} 3(\mathrm{w} 3 \mathrm{f} 2(\mathrm{w} 2 \mathrm{f} 1(\mathrm{w} 1 \mathrm{p}+\mathrm{b} 1)+\mathrm{b} 2)+\mathrm{b} 3)$ (2)

The output of layers one and two are the inputs to the layers two and three. The third layer is the output layer and the layers one and two are the called as the hidden layers [15].

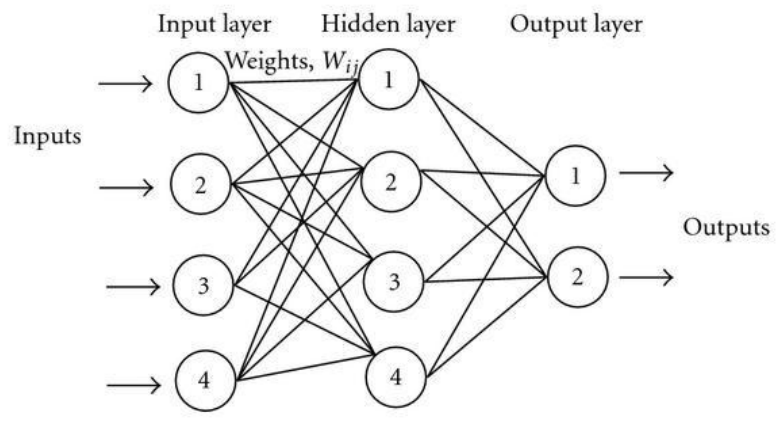

Fig.1 Multilayer Neural Network

\section{DISSCUSIONS}

Though the first description of Parkinson's disease (PD) was given by James Parkinson in early $19^{\text {th }}$ century. The knowledge of the PD has been present in India since ancient times. The Prevalence of PD is less compared to other countries. PD has been known in India since ancient days and the powder of Mucuna Pruriens seeds was used for its treatment. The present day management of PD in India is similar to that in the other countries. [16]

A study of 92 research papers about PD in India, Non motor symptoms and genetics aspects are used for PD diagnosis. Most of the genetic mutations found to cause PD in other population are not found in India. There need to be more studies on therapeutic aspects of the disease. The study about the literature of PD in India are very less. The review shows that people of India may differ from rest in the context of PD [17]. The present day management of PD in India is similar to that in the other countries [16].

Among various study about the PD diagnosis through Artificial Neural Networks are discussed below.

Information Gain is used to reduce the number of attributes twenty two to sixteen using the Artificial Neural Networks. The accuracy obtained in this 
technique are $82.051 \%$ in training the dataset and $83.333 \%$ in validating the dataset [18]. Another work done with this Parkinson's Dataset classification using Artificial Neural Network. The classifiers used are Random Tree, Support Vector Machine and Feedforward Back-propagation based Artificial Neural Networks. Through this method the accuracy got are $97.37 \%$ [19]. Acoustic voice analysis and measurement is also used for diagnosis neurological disordered voices like Parkinson's Disease. In this method they uses Mel-frequency cepstral coefficient features. They used 137 sustained vowel phonations among 73 patients were suffering from neurological disorders and 64 were healthy peoples includes both male and female. Artificial Neural Network were used for classification of neurological disorders and healthy people. The classification accuracy they obtained are $92 \%$ [20].

Cartesian Genetic Programming evolved Artificial Neural Network is applied for diagnosing Parkinson's Disease. The experiment analysis is in progress by them using Genetic Programming method [21]. Four Classification Methods are used for diagnosing Parkinson's Disease. These four classification schemes are Neural Networks, DMneural, Regression and Decision Tree. Among these four methods Neural Networks yield better results of about 92.9\% [22]. The previous method with the accuracy of $92.9 \%$ [22] comes with a cost of reduced prediction accuracy of the small class. The Neural Network is designed to boost by filtering. It results in increase of robustness and obtained the accuracy of $>90 \%$ [23]. Also the selected fields 1-17-19-20 produces the highest accuracy compared the other methods. SVM produces $91.40 \%$, MLPNN produces $89.90 \%$, RBFNN produces $87.63 \%$, ANFC-LH produces $94.72 \%$ accuracy in testing [24]. In the above mentioned methods the attribute values chosen are 2-17-19-21.

Another classification diagnosis of PD using Genetic Algorithm and SVM indicates that the classification accuracy achieved by them are $94.50 \%$ with the attributes Fhi $(\mathrm{Hz})$, Fho $(\mathrm{Hz})$, jitter (RAP) and shimmer (APQ5). With selection of 7 attributes Fhi (Hz), Fho (Hz), Flo (hz), jitter (RAP), shimmer (APQ5), Jitter (ABS), shimmer they obtained the accuracy of $93.66 \%$ is obtained. With selection of 9 attributes Fhi $(\mathrm{Hz})$, Fho (Hz), Flo (hz), jitter (RAP), shimmer (APQ5), Jitter(ABS), shimmer, Jitter (\%), HNR 94.22\% accuracy is obtained using SVM classifier [25].

\section{LEARNING METHODS}

A Machine Learning research majorly focus to automatically learn and recognize complex patterns and make intelligent decisions based on the data. Machine Learning must adopt to the human learning methods like [26].

\section{Perceptual Learning}

The learning of new objects, categories, relations etc.,

\section{Episodic Learning}

Learning of events like what, when and where.

\section{Procedural Learning}

The learning of new actions and action sequences with which to accomplish new tasks. Machine Learning algorithms have proven to be of great practical value in many applications.

Common Machine Learning types are:

\section{1) Supervised Learning:}

where the algorithm generates a function that maps inputs to desired outputs. One standard formulation of the supervised learning task is the classification problem: the learner is required to learn (to approximate the behavior of) a function which maps a vector into one of several classes by looking at several input-output examples of the function.

objective of specialization is to obtain the whole atlas of specialization chains (graphs) by assigning various types of members and joints to each available generalized chain (graphs) subject to the design requirements and design constraints specified above.

\section{2) Unsupervised Learning}

which models a set of inputs: labeled examples are not available.

\section{3) Semi Supervised Learning}

Which combines both labeled and unlabeled examples to generate an appropriate function or classifier.

\section{4) Reinforcement Learning}

Where the algorithm learns a policy of how to act given an observation of the world. Every action has some impact in the environment, and the environment provides feedback that guides the learning algorithm.

\section{5) Transduction}

Is similar to supervised learning, but does not explicitly construct a function: instead, tries to predict new outputs based on training inputs, training outputs, and new inputs [26].

\section{BACK PROPOGATION}

Backpropagation was created to multi-layer networks and nonlinear differentiable transfer functions. It is a common method used in ANN for training. When an input is given to the network it traverses through layer by layer until it reaches the output. The output is compared with the desired output using the loss 
function. Error value is calculated in each neurons and propagates backward until each neuron has an associated error value nearer to the desired output [27].

This training method updates the weights and bias values according to Levenberg-Marquardt optimization. It can train any network if it is provided with weight input and transfer functions have derivative functions.

It requires more memory and is the fastest backpropagation algorithm which is mostly used for supervised algorithm [28].

So trainlm method is used for the training of the PD dataset to get more accurate and fast result.

\section{EXPERIMENTAL RESULTS}

From the 23 attributes of the PD dataset to select the desired attribute for training with ANN, classification process is done with each attribute with a non-linear measurement of fundamental frequency variation ie., Spread1 and Status column is chosen. 18 graphs are plotted to visualize the better classification values to predict PD and healthy persons. Another set of 18 graphs were plotted for better classification of each field with non-linear measurement of fundamental frequency variation ie., Spread2 and Status column. Totally 36 graphs are plotted for identifying better classification values present in the dataset to predict the PD or healthy persons.

The fields chosen from PD dataset are based on less percentage of overlaps produced by the classification graphs. Among 36 classification graphs the less overlap fields found are the set1: MDVP:Fo(Hz), Spread1 and Status, set2: DFA, Spread1 and Status, Set3: MDVP:Fo(Hz), Spread2 and Status, Set4: Shimmer:APQ3 ,Spread2 and Status. So these four sets are chosen for training and testing. In the first of classification, each column with Spread1 and Status, the most accurate classification occurs for the field of MDVP:Fo(Hz), RPDE and DFA respectively.

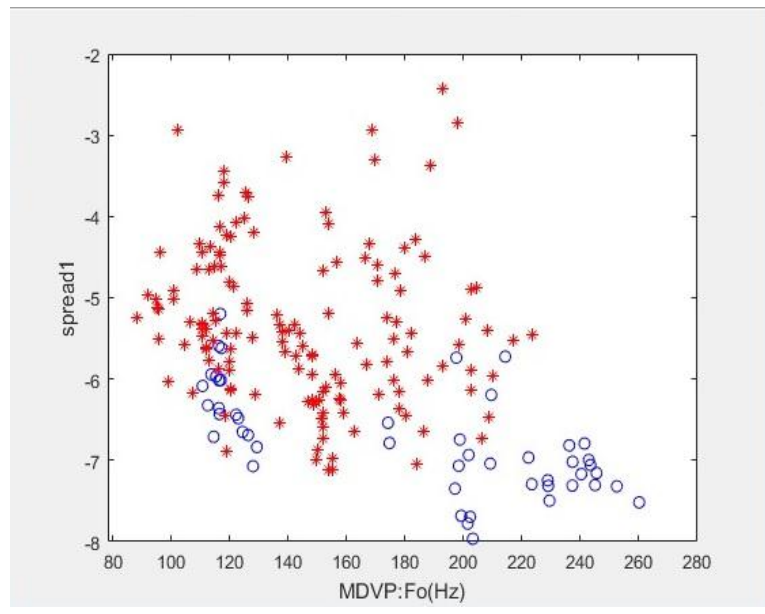

Fig. 2 Graph of MDVP: Fo(Hz), Spread1 Vs Status

Fig. 2 shows the classification for the values associated with the attributes MDVP: $\mathrm{Fo}(\mathrm{Hz})$ (Average Vocal
Fundamental Frequency), Spread1(Non-Linear measures of Fundamental Frequency Variation) and Status.

Fig.3 shows the classification for the values associated with the attributes DFA (Signal Fractal Scaling Exponent), Spread1(Non-Linear measures of Fundamental Frequency Variation) and Status.

Among the classification of each 23 columns with Spread1 and Status; MDVP: Fo(Hz), Spread1 and Status has the accurate classified values without much overlapping and it is followed by DFA, Spread1 and Status as shown in Fig.3. So, the fields chosen for the training through ANN are MDVP: $\mathrm{Fo}(\mathrm{Hz})$, DFA, Spread1 and Status (1-17-19-20).

Among 195 variant values of 31 people are taken for training and testing. About $75 \%$ of data is assigned for training and $25 \%$ of data is assigned for testing. Levenberg-Marquart optimization is used. Network Type used are: Feed Forward Backpropagation, the Training Function used are TRAINLM, Adaptive Learning Function used are: LEARNGDM and the Transfer Function used are TRANSIG. The output obtained is shown in the Regression Graph. Fig.4.

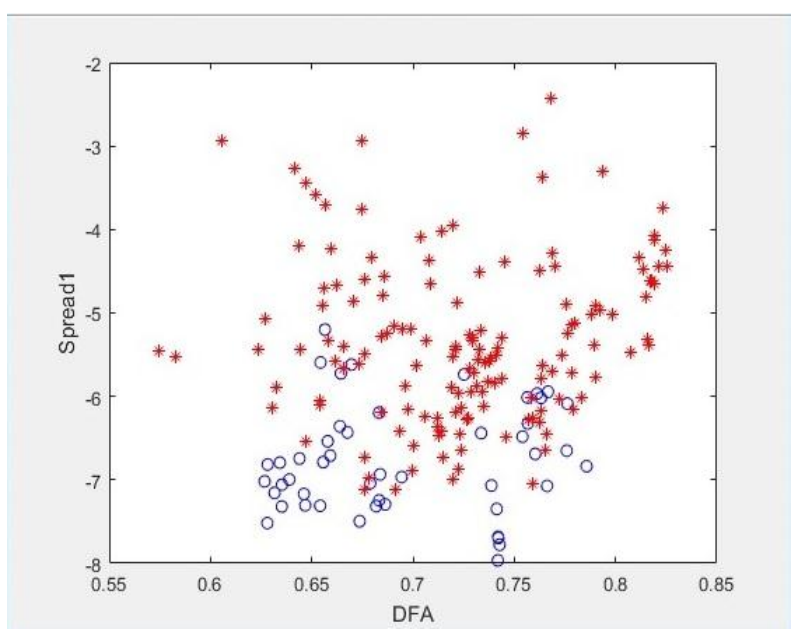

Fig. 3 Graph of DFA, Spread1 Vs Status
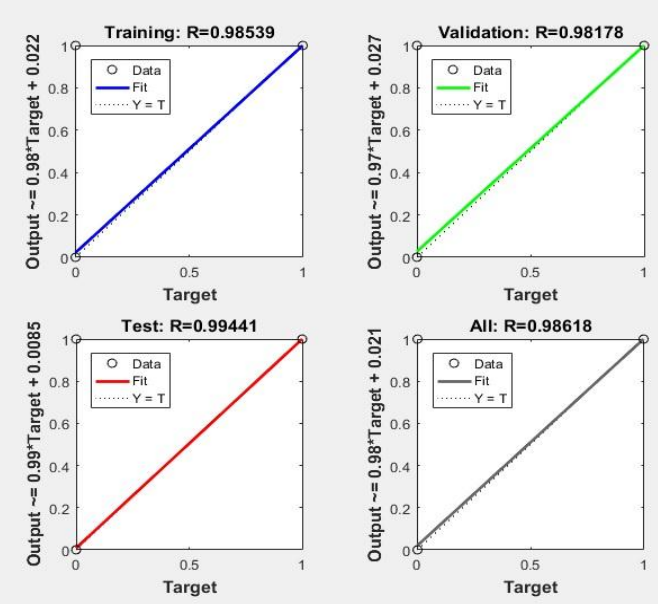

Fig. 4 Regression graph of MDVP: Fo(Hz), DFA, Spread1 and Status 
In Fig. 4 shows the training and testing results of the four parameters MDVP: Fo(Hz), DFA, Spread1, Status using the trainlm function.

Table 2 Resultset of 1-17-19-20 attributes

\begin{tabular}{|c|c|c|c|}
\hline Attributes & Method & $\begin{array}{c}\text { Training } \\
\text { \% }\end{array}$ & Testing \% \\
\hline $1-17-19-20$ & FFBP & 98.53 & 99.44 \\
\hline
\end{tabular}

In the next set of classification is done for each column with Spread2 and Status and the most desirable classification occurs for the field of MDVP: $\mathrm{Fo}(\mathrm{Hz})$, Spread2, and Status. And the next desirable classification occurs for the attributes Shimmer: APQ3, Spread2 and Status.

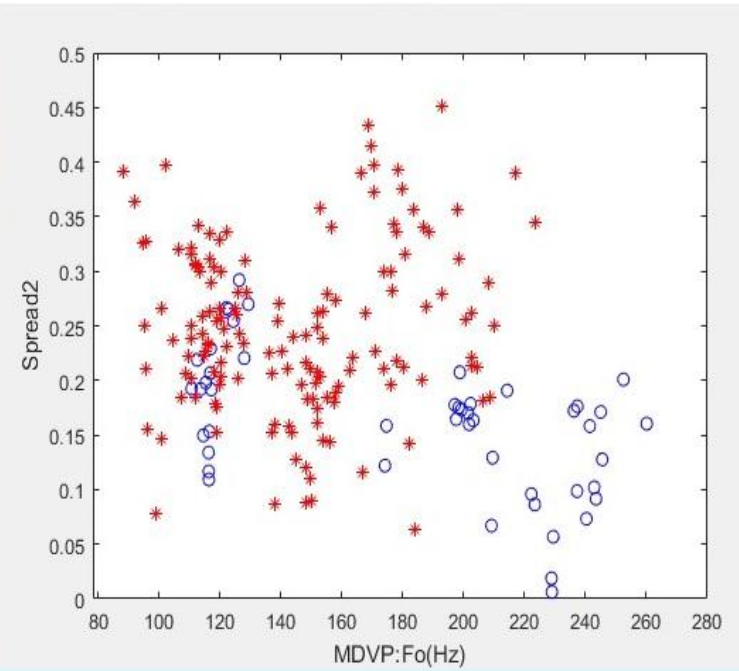

Fig. 5 Graph of MDVP: Fo(Hz), Spread2 vs Status

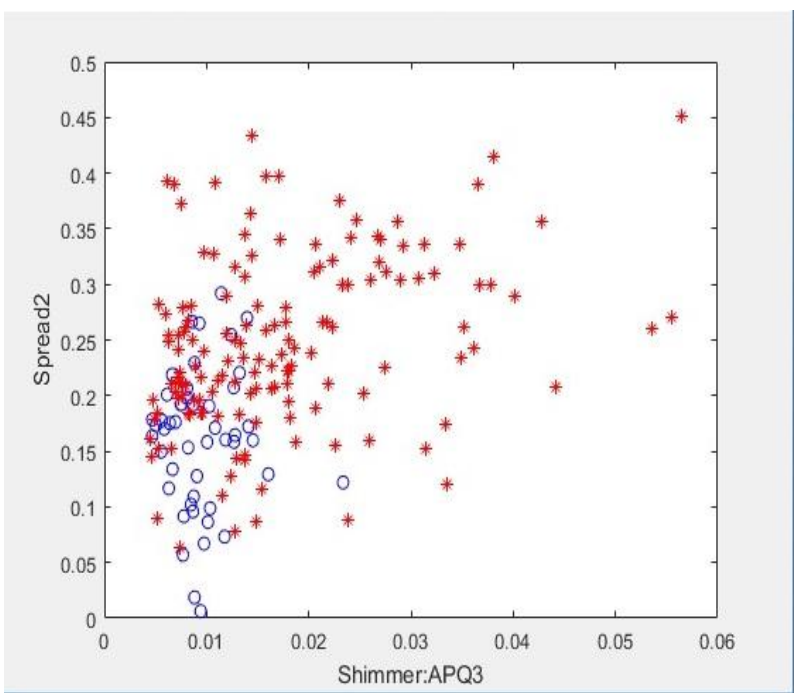

Fig. 6 Graph of Shimmer: APQ3, Spread2 Vs Status

Another set of classification for 23 columns with Spread2 and Status is done. Among this MDVP: Fo(Hz), Spread2 and Status has the clear classified values without much overlapping and it is followed by Shimmer: APQ3, Spread2. and Status as shown in
Fig.5. So, the fields chosen for the training of ANN are MDVP: Fo(Hz), Spread2 and Status. (1-11-17-21)

Among 195 variant values of 31 people are taken for training and testing. About $75 \%$ of data is assigned for training and $25 \%$ of data is assigned for testing. Levenberg-Marquart optimization is used. Network Type used are Feed Forward Backpropagation, the Training Function used are TRAINLM, Adaptive Learning Function used are LEARNGDM and the Transfer Function used are TRANSIG.

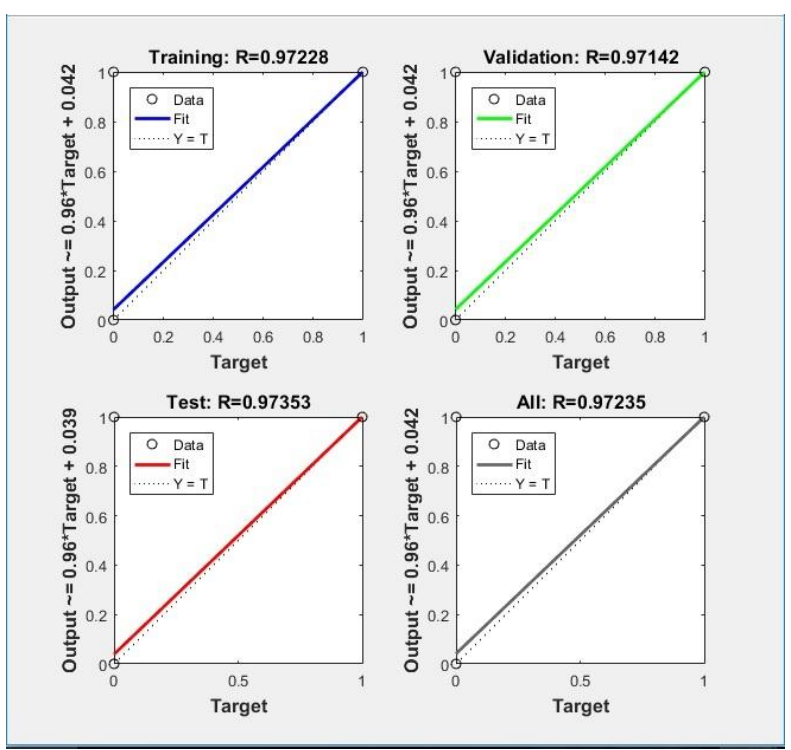

Fig. 7 Regression graph of MDVP: Fo(Hz), Shimmer: APQ3, Spread2 and Status

In Fig. 7 shows the training and testing results of the four parameters MDVP: $\mathrm{Fo}(\mathrm{Hz})$, Shimmer: APQ3, Spread 2 and Status using the trainlm function.

Table 3 Resultset of 1-11-17-21 Attributes

\begin{tabular}{|c|c|c|c|}
\hline Attributes & Method & $\begin{array}{c}\text { Training } \\
\mathbf{\%}\end{array}$ & Testing \% \\
\hline $1-11-17-21$ & FFBP & 97.22 & 97.35 \\
\hline
\end{tabular}

\section{CONCLUSIONS}

Experimental result shows that feed forward backpropagation of ANN with trainlm method has highest accuracy of training and testing values $98.53 \%$ and $99.44 \%$ respectively with the highest classified attribute values of 1-17-19-20 compared to the attribute values 1-11-17-21 which score $97.22 \%$ in training and $97.35 \%$ in testing. Number of Layers used are 8 . Number of neurons, hidden layers used for experimenting are 10 . This experiment with different field and method with trainlm function produces more accurate results which helps in classifying PD affected people with the healthy people. Hence it is more useful for diagnosing the PD affected people. 


\section{ACKNOWLEDGMENT}

The dataset was created by Max Little of the University of Oxford in collaboration with the National Centre for Voice and Speech, Denver, Colorado. We thank for all. I thank for my parent's A.Dhandapani and G.Yogamangalam for their endless support to my work.

\section{REFERENCES}

[1] http://www.parkinson.org/understandingparkinsons/ what-is-parkinsons

[2] Gelb, D., Oliver, E., Gilman S, "Diagnostic criteria for Parkinson disease". Arch Neurol 56 (1): 33-9, doi:10.1001/archneur.56.1.33. PMID 9923759, 1999.

[3] http://www.parkinson.org/understandingparkinsons/what-is-parkinsons/The-Stages-ofParkinsons-Disease.

[4] Pezard, L., Jech, R. and RuÊzzlicÏka, E. (2001) Investigation of Non-Linear Properties of Multichannel EEG in the Early Stages of Parkinson's Disease. Clinical Neurophysiology, 122, 38-45

[5] Ene, M. (2008) Neural Network-Based Approach to Discriminate Healthy People from Those with

Parkinson's Disease. Mathematics and Computer Science Series, 35, 112-116.

[6] Little, M.A., McSharry, P.E., Hunter, E.J., Spielman, J. and Ramig, L.O. (2008) Suitability of Dysphonia Measurements for Telemonitoring of Parkinson's Disease. IEEE Transactions on Biomedical Engineering,56,1015-1022. http://dx.doi.org/10.1109/TBME. 2008.2005954.

[7] Caglar, M.F., Cetisli, B. and Toprak, I.B. (2010) Automatic Recognition of Parkinson's Disease from Sustained Phonation Tests Using ANN and AdaptiveNeuro-Fuzzy Classifier. Journal of Engineering Science Design, 1, 59-64

[8] Gil, D. and Johnson, M. (2009) Diagnosing Parkinson by Using Artificial Neural Networks and Support Vector Machines.Global Journal of Compute Science and Technology, 9, 63-71

[9] Duffy, R.J. (2005) Motor Speech Disorders: Substrates, Differential Diagnosis and Management. 2nd Edition, Elsevier Mosby, St. Louis.

[10] Ho, A.K., Iansek, R., Marigliani, C., Bradshaw, J.L. and Gates, S. (1998) Speech Impairment in a Large Sample of Patients with Parkinson's Disease. Behaviour Neurology,11,131-137. http://dx.doi.org/10.1155/1999/327643.

[11] Sapir, S., Spielman, J.L., Ramig, L.O., Story, B.H. and Fox, C. (2007) Effects of Intensive Voice Treatment (the Lee Silverman Voice Treatment [LSVT]) on Vowel Articulation in Dysarthric Individuals with Idiopathic Parkinson Disease: Acoustic and Perceptual Findings.Journal of Speech Lang Hearing Research, 50, 899-912.
[13] Rahn, D.A., Chou, M., Jiang, J.J. and Zhang, Y. (2007) Phonatory Impairment in Parkinson's Disease: Evidence from Nonlinear Dynamic Analysis and Perturbation Analysis. Journal of Voice, 21, 64-71

[14] Center for Machine Learning and Intelligent Systems, 2008, http://archive.ics.uci.edu/ml/datasets/Parkinsons.

[15] Eldon Y. Li, "Artificial Neural Networks and their Business Applications”, Taiwan, 1994. FLEXChip Signal Processor (MC68175/D), Motorola, 1996

[16] Epidemiology and treatment of Parkinson's disease in India, Singhal B, Lalkaka J, Sankhla C.Pubmed, 2003.

[17] Research in Parkinson's disease in India: A review, Pratibha Surathi, Ketan Jhunjhunwala, Ravi Yadav, and Pramod Kumar Pal, Pubmed,2016.

[18] Parkinson's Disease Classification using Neural Network and Feature selection, Anchana Khemphila and Veera Boonjing, World Academy of Science, Engineering and Technology International Journal of Mathematical, Computational, Physical, Electrical and Computer Engineering Vol:6, No:4, 2012.

[19] Performance comparison of heterogeneous classifiers for detection of Parkinson's disease using voice disorder(dysphonia),International Conference on Informatics, Electronics \& Vision (ICIEV), 2014, Mohammad S.Islam ; Imtiaz Parvez; Hai Deng ; Parijat Goswami.

[20] Automatic detection of neurological disordered voices using mel cepstral coefficients and neural networks, Uma Rani K ; Mallikarjun S. Holi, Point-of-Care Healthcare Technologies (PHT), 2013 IEEE.

[21] Bio-signal Processing Using Cartesian Genetic Programming Evolved Artificial Neural Network (CGPANN), Arbab Masood Ahmad ; Gul Muhammad Khan, 10th International Conference on Frontiers of Information Technology(FIT), 2012.

[22] R. Das, A comparison of multiple classification methods for diagnosis of Parkinson disease, Expert Systems with Applications 37 (2) (2010) $1568-1572$.

[23] Neural Networks to Diagnose the Parkinson's Disease,scjournal.ius.edu.ba/index.php/scjournal/ article/viewFile/48/48,2013.

[24] Automatic Recognition of Parkinson's Disease from sustained Phonation Tests Using ANN and Adaptive Neuro-Fuzzy Classifier.,Journal of Engineering Science and Design vol. 1,No.2, pp.59-64,2010

[25] Shahbakhi, M., Far, D.T. and Tahami, E. (2014) Speech Analysis for Diagnosis of Parkinson's Disease Using Genetic Algorithm and Support Vector Machine. J. Biomedical Science and Engineering, 7, 147-156. 
[26] F. Lancer, D. Eilers, and R. Knorr. Fault deduction in discrete event based distributed systems by forecasting message sequences with neural networks. In KI 2009: Advances in Artificial neuralnetworks. In KI 2009: $\quad$ Advances in Artificial Intelligence, Volume 5803

[27] Neural Network Design, by T. Hagen, B. Demuth, Beale and De Jesus.

[28] https://in.mathworks.com/help/nnet/ref/ trainlm.html 